Revista de

Contabilidade e

Organizações

www.rco.usp.br
DOI: http://dx.doi.org/10.11606/issn.1982-6486.rco.2020.169560

Journal of

Accounting and

Organizations

\title{
Uso corporativo de mídias sociais e o desempenho de mercado
}

\author{
Corporate use of social media and market performance
}

Mikaéli da Silva Giordani ${ }^{a}$, Micheli Aparecida Lunardia, Roberto Carlos Klann ${ }^{\text {a }}$

${ }^{a}$ Fundação Universidade Regional de Blumenau

Palavras-chave

Mídias sociais.

Desempenho organizacional.

Empresas brasileiras.

Twitter.

\section{Resumo}

As empresas estão sendo impulsionadas a usar as mídias sociais, entretanto, pouco se sabe sobre o impacto do uso de tais mídias no seu desempenho. Esta pesquisa objetivou verificar a relação entre o uso corporativo de mídias sociais e o desempenho de mercado de empresas brasileiras. Para isso utilizou-se regressão linear múltipla (OLS). O período de análise compreendeu os anos de 2013 a 2017 e a amostra constituiu-se de 225 empresas. O uso das mídias sociais correspondeu a utilização do Twitter para publicação de informações financeiras, enquanto o desempenho de mercado foi mensurado pelo retorno anormal das ações. Os resultados revelaram que o uso corporativo das mídias sociais apresenta relação positiva com o desempenho. Conclui-se que a publicação de tweets sobre informações financeiras pode maximizar os retornos, o que indica que as mídias sociais podem se configurar em mais uma fonte de informações para stakeholders. Como contribuições, os achados evidenciam benefícios reais obtidos com o uso das mídias sociais, ao demonstrar que o uso corporativo do Twitter influencia os participantes do mercado de capitais e, consequentemente, melhora o desempenho de mercado da organização. Tal evidência pode motivar a alta gerência das empresas a fornecer suporte inicial e contínuo para o uso de mídias sociais nas organizações.

\begin{abstract}
Companies are being driven to use social media, however little is known about the impact of using these media on their performance. This study seeks to verify the relationship between the corporate use of social media and the market performance of Brazilian companies. To accomplish this, we will use ordinary least square (OLS) multiple linear regressions. The analysis period covers the years 2013 to 2017, and the sample consists of 225 companies. The use of social media corresponds to the use of Twitter for publishing financial information, while market performance was measured by abnormal returns on shares. The results reveal that the corporate use of social media has a positive relationship with performance. We conclude that the publication of tweets about financial information can maximize returns, which indicates that social media can be another source of information for stakeholders. This study contributes to the literature by showing real benefits obtained with the use of social media, and by demonstrating that the corporate use of Twitter influences capital market participants and, as a result, improves the organization's market performance. This evidence may motivate upper level corporate management to provide initial and continuous support for the use of social media.
\end{abstract}

\section{Implicações práticas}

O estudo apresenta os efeitos das mídias sociais no desempenho de mercado de empresas brasileiras. Essa análise auxilia a compreensão de como publicações financeiras em mídia social podem ser benéficas para as organizações, ao reduzir a assimetria de informações entre empresa e investidores e analistas, de modo a maximizar retornos. Tal evidência pode motivar gestores e investidores a utilizar esses canais.

Copyright (C) 2020 FEA-RP/USP. Todos os direitos reservados 


\section{INTRODUÇÃO}

A adoção das mídias sociais na sociedade tem levado as organizações a adotarem ferramentas tecnológicas para divulgar e comunicar informações organizacionais importantes às partes interessadas na organização (Smits \& Mogos, 2013; Kim \& Youm, 2017). Segundo Lee, Hutton e Shu (2015), elas se tornaram um canal viável para divulgação de informações importantes, quando comparadas aos canais tradicionais de informação, possuem maior alcance e permitem que as empresas enviem de forma direta e com rapidez a mensagem pretendida.

A mídia social permite que indivíduos e comunidades se comuniquem e divulguem informações com menor custo e de forma instantânea na web, com um alcance potencialmente global. Tendo em vista que o mercado de capitais é caracterizado por negociações de alta frequência e mudanças na formação de preços, o uso de tecnologias nesse processo afetou a forma como os mercados de capitais processam e respondem às notícias sobre as empresas (Miller \& Skinner, 2015).

As mídias sociais foram utilizadas pelos gestores no princípio para fins de marketing (Arnaboldi, Busco, \& Cuganesan, 2017). Posteriormente, empresas passaram a usar essas ferramentas principalmente para transmitir a imagem de como eles desejam que sua empresa seja vista (Harquail, 2011). Esse meio de divulgação vem ganhando popularidade e sendo cada vez mais utilizado em operações regulares de muitas empresas (Smits \& Mogos, 2013). Aquelas que utilizam as tecnologias mais recentes de mídia social parecem superar seus concorrentes e relatam alguns benefícios, como custos mais baixos e melhor eficiência (Harris \& Rea, 2009). Portanto, é importante entender a relação entre o uso das mídias sociais e o desempenho das empresas (Smits \& Mogos, 2013; Wetzstein, Leitner, Rosenberg, Dustdar \& Leymann, 2011).

As mídias sociais envolvem um conjunto de informações provenientes de diferentes usuários, como empresas, acionistas e investidores, as quais podem levar a mudanças no mercado de capitais (Arias, Arratia \& Xuriguera, 2014). Conforme Zhang (2015), as mídias socias englobam públicos maiores e, consequentemente, influenciam mais investidores e proporcionam melhores retornos aos acionistas.

No entanto, no cenário brasileiro, pouco se sabe sobre o reflexo do uso das mídias sociais no desempenho empresarial. Segundo Paniagua e Sapena (2014) e Arnaboldi et al. (2017), estudos a respeito dessa temática carecem de maior atenção, afinal, o espaço da mídia social se tornou um lugar comum para comunicação, estabelecimento de redes e compartilhamento de conteúdo. Exemplos de tweets que repercutiram e causaram efeitos de imediato na empresa foram os publicados pela empresa Via Varejo e pelo CEO da empresa Tesla, Elon Musk. A empresa Via Varejo publicou tweets com números elevados de vendas da varejista, fato que impulsionou o valor das ações em mais de 7\%. Elon Musk tweetou que os papeis da empresa estavam supervalorizados, o que fez com que o valor das ações imediatamente caíssem cerca de 10\%. Esses fatos apresentam indícios sobre os possíveis efeitos do uso dessas ferramentas no meio corporativo. Entretanto, são casos isolados, que repercutem além das mídias sociais porque se tornam alvos de investigação. Diante disso, verifica-se a importância de compreender a atuação das empresas nas mídias sociais e os reflexos dessa ação.

Desta maneira, apresenta-se a seguinte questão-problema: Qual a relação entre o uso corporativo das mídias sociais e o desempenho de mercado de empresas brasileiras? Assim, o objetivo do estudo é investigar se a utilização de mídias sociais por parte de empresas, para divulgação de informações financeiras, pode melhorar seu desempenho de mercado.

O estudo se justifica pela importância em compreender o comportamento das empresas no ambiente de mídias sociais e a forma como estas influenciam os participantes do mercado e afetam o valor da empresa (Jung, Naughton, Tahoun, \& Wang, 2018; Kim \& Youm, 2017, Zhang, 2015). A mídia social têm sido uma nova vertente importante da literatura, dada a sua crescente utilização pela sociedade e o potencial para os usuários criarem e disseminarem seu próprio conteúdo sobre as empresas. Isso representa uma nova questão na literatura, porque as plataformas prévias de divulgação impediam, ou, pelo menos, limitavam a medida que os investidores, em geral, poderiam responder e divulgar seus próprios pontos de vista sobre as empresas (Jung et al. 2018; Miller \& Skinner, 2015).

$\mathrm{Na}$ atualidade, as mídias sociais tornaram-se onipresentes e estão desempenhando um papel cada vez mais crítico nos ambientes de negócios atuais. As novas possibilidades informacionais que elas apresentam podem afetar e impactar as relações entre clientes e gestores das organizações. Agostino e Sidorova (2017) destacam que quando a relação entre cliente e organização fica desfocada, as mídias sociais contribuem para a remodelação do relacionamento entre cliente e organização. 
Yu, Duan e Cao (2013) demonstraram que o desempenho de mercado tem relação positiva com as mídias sociais e que tweets positivos contribuem para o aumento no retorno das ações, enquanto tweets negativos impactam negativamente. Este estudo se diferencia da pesquisa de Yu et al. (2013) ao analisar palavras relacionadas diretamente ao desempenho das empresas, enquanto aquele utilizou análise de sentimento. Além disso, este estudo contempla o período de 2013 a 2017, enquanto o anterior analisou três meses do ano de 2011. Além da diferença temporal, Yu et al. (2013) analisaram empresas no mercado norte-americano, enquanto neste estudo o foco foi em empresas brasileiras.

Acredita-se que além da diferença em termos de tamanho e de liquidez entre os dois mercados, há também diferenças em termos de uso das mídias sociais para fins corporativos entre as empresas dos dois países. Portando, apesar do estudo supracitado ter realizado uma análise mais restrita em termos temporais, mas mais profunda em termos de mídias sociais analisadas, pois considerou publicações em $b \log s$, fóruns e publicações convencionais, como o Google News, este estudo avança ao fazer uma análise sobre o mercado brasileiro, que cobre um período mais recente.

Este estudo contribui de diferentes maneiras para a pesquisa contábil. Inicialmente, contribui para a literatura que investiga o comportamento de divulgação de informações das empresas (Jung et al., 2018). Além disso, Paniagua e Sapena (2014) destacam que estudos sobre mídias sociais e o desempenho organizacional podem contribuir para os gestores das organizações, bem como para os consultores financeiros, por meio da análise das informações disponíveis. Ainda, a pesquisa contribui para a compreensão de como os gerentes atuam no ambiente de informação global das mídias sociais de uma empresa, de forma a influenciar externamente as decisões de investidores e internamente as mudanças de valores contábeis da empresa.

\section{ANTECEDENTES E HIPÓTESE DE PESQUISA}

O termo mídia social refere-se à prática de usar qualquer meio específico socialmente, também, é comumente utilizado para descrever aplicativos da web, como sites de redes sociais e plataformas de compartilhamento de conteúdo gerado por usuários (Kaplan \& Haenlein, 2010). Kaplan e Haenlein (2010) destacam que a mídia social refere-se a plataformas dinâmicas de internet e sua capacidade de apoiar a interatividade e a comunicação em tempo real. Para Arnaboldi et al. (2017) e Scott e Orlikowski (2009), caracterizam-se como tecnologias, utilizadas pelas organizações para criar, comunicar e trocar informações em comunidades e redes virtuais.

As mídias sociais e, em particular, o Twitter, oferecem um cenário único para examinar se as empresas se disseminam estrategicamente. Se uma empresa quer publicitar informações relacionadas ao investidor, como um anúncio de ganhos, faria isso enviando um comunicado à imprensa (Frankel, Johnson \& Skinner, 1999). Sob esta abordagem, uma empresa não saberia quando algum dos seus investidores existentes ou potenciais receberia a informação. No entanto, uma empresa pode usar o Twitter para divulgar diretamente informações aos seus seguidores, controlar o tempo de disseminação, enviar mensagens repetidas durante dias, relacionadas ao mesmo evento e saber o número exato de seguidores (Jung et al., 2018). Além disso, as redes sociais, muitas vezes, incentivam a transparência das informações da empresa (Bonson \& Ratkai, 2013) e possibilitam minimizar informações negativas (Jung et al., 2018; Lee et al., 2015).

Conforme Zhang (2015), empresas que são altamente ativas na adoção de mídias sociais para divulgação, tendem a usá-las para intensificar a divulgação de informações. Nesse sentido, o seu uso intensivo para divulgação por parte das empresas, bem como a divulgação voluntária de informações nesse meio são impulsionadas por fatores, como: desempenho da empresa, ambiente de informação, nível de assimetria de informações e estrutura da dívida (Zhang, 2015).

Como uma plataforma de comunicação baseada na internet, as mídias sociais oferecem recursos imediatos e permitem que os usuários troquem informações em tempo hábil, busquem e forneçam feedback rápido por meio de posts e diálogo em tempo real (Lodhia e Stone, 2017). Segundo o estudo de Smits e Mogos (2013), a combinação de mídias sociais interligadas forma um sistema que aprimora a capacidade dos negócios. Além disso, as evidências empíricas demonstram que o seu uso corporativo aumenta a capacidade e desenvolvimento do negócio (Cao, Aijan, Hong, \& Le, 2018; Parveen, Jaafar, \& Ainin, 2016; Arnaboldi \& Coget, 2016; Jaafar \& Shuib, 2015).

O uso corporativo das mídias sociais foi explorado em estudos com a finalidade de evidenciar a sua relação com o desempenho organizacional. Rodriguez, Peterson e Ajjan (2015) relataram que tais tecnologias impactam positivamente o desempenho de vendas de uma organização. A utilização de mídias sociais ajuda as empresas a apoiarem e criarem um relacionamento com os clientes (He, Wang, \& Akula, 2017; Agostino \& Sidorova, 2017; Ainin et al., 2015). 
Dessa forma, as informações fornecidas nessas plataformas possibilitam que as empresas se concentrem nas necessidades e preocupações dos clientes, tornando-se mais competitivas, o que, consequentemente, resulta em um melhor desempenho organizacional (Rodriguez et al., 2015).

Ferrer, Bousoño, Jorge, Lora, Miranda e Natalizio (2013) demonstraram que o uso de tecnologias de mídia social impacta positivamente o capital social de uma organização e, portanto, no seu desempenho. A inclusão de redes sociais na organização facilita a ligação entre seus membros e reduz a distância organizacional entre gerentes e funcionários, fazendo com que os últimos se sintam parte do processo de tomada de decisão da empresa, o que aumenta sua motivação e influencia no desempenho organizacional (Ferrer et al., 2013).

Estudos mostraram que o uso desta tecnologia pode melhorar os processos de negócios (Paniagua \& Sapena, 2014). Alguns pesquisadores descobriram que a sua adoção corporativa proporciona benefícios e identificaram uma relação positiva entre a adoção de mídia social e o desempenho corporativo (Parveen et al., 2016; Ainin et al., 2015, Paniagua \& Sapena, 2014).

Ainin et al. (2015) identificaram que o uso de mídia social tem relação positiva com o desempenho econômico e, da mesma forma, verificou-se também que o seu uso impacta positivamente o desempenho não financeiro, em termos de redução de custos em marketing.

Diante desse contexto, acredita-se que as empresas utilizem as mídias sociais para divulgar informações positivas a seu respeito e, com isso, melhorar seu desempenho de mercado. A partir disso, elaborou-se a seguinte hipótese de pesquisa:

$\mathbf{H}_{1}$ : $\mathrm{O}$ uso corporativo de mídias sociais está relacionado positivamente ao desempenho de mercado de empresas brasileiras.

\section{MÉTODO E PROCEDIMENTOS DA PESQUISA}

A população desta pesquisa é composta pelas companhias abertas listadas na B3 (Brasil, Bolsa e Balcão). Para a amostra foram selecionadas apenas as companhias que apresentaram dados para o cálculo da variável dependente e variáveis de controle. Após, foi observado se as organizações possuíam mídia social (Twitter) e, posteriormente, as que divulgavam informações financeiras no Twitter. Justifica-se a utilização do Twitter como mídia social no presente estudo, pois, conforme Jung et al. (2018), esta corresponde a uma das plataformas de mídia social mais usadas para fins corporativos.

O procedimento seguiu a metodologia abordada por Jung et al. (2018). Dessa forma, primeiramente, verificou-se o website corporativo de cada empresa que compõe a amostra para evidenciar se ele possuía link que direcionasse à mídia social. Esse procedimento é fundamental, pois, garante que a mídia social acessada seja a oficial da empresa. Posteriormente, foi acessada a mídia social para confirmar sua existência e identificar a divulgação de informações financeiras.

Detalhadamente, o procedimento consistiu em carregar todos os tweets publicados na página de cada empresa até o início do período analisado (2013) e, posteriormente, realizar filtros. Para isso, com base no estudo de Jung et al. (2018), os filtros e procuras foram realizados com as seguintes palavras-chave: ganhos, lucros, receita, resultados, trimestre, lucro por ação e crescimento. Ressalta-se que nesta pesquisa, quando se trata de divulgação de informações financeiras no Twitter, refere-se à divulgação de informações com os termos acima citados.

Dessa maneira, a amostra constituiu-se de 225 empresas, sendo que destas 23 empresas utilizaram o Twitter para divulgar informações financeiras. O período de análise compreendeu os anos de 2013 a 2017 , resultando em 1.125 observações. A Tabela 1 apresenta o número de empresas que divulgaram informações e o número de publicações realizadas, em cada ano de analise.

Tabela 1. Amostra da pesquisa por setor de atuação e quantidade de empresa ano com Twitter

\begin{tabular}{lccccc}
\hline Itens & $\mathbf{2 0 1 3}$ & $\mathbf{2 0 1 4}$ & $\mathbf{2 0 1 5}$ & $\mathbf{2 0 1 6}$ & $\mathbf{2 0 1 7}$ \\
\hline Empresas no Twitter & 5 & 9 & 12 & 14 & 19 \\
Número publicações no Twitter (tweets) & 19 & 37 & 58 & 153 & 210 \\
\hline
\end{tabular}

Fonte: dados da pesquisa. 
Verifica-se na Tabela 1 que tanto o número de empresas quanto de publicações apresentou um aumento no decorrer dos anos. No ano de $2017,82 \%$ das empresas que divulgaram informações financeiras apresentaram publicações nesse ano. Além disso, em 2017 foram publicadas cerca de 44\% das informações financeiras referentes ao período analisado. Esssas evidências demonstram o aumento da utilização do Twitter como uma ferramenta corporativa para divulgar informações financeiras, especificamente observa-se um aumento de mais de 10 vezes em relação ao início do período (2013).

Utilizou-se neste estudo a variável retorno anormal (DES) como variável dependente, a qual corresponde ao desempenho de mercado, tweets referentes a informações financeiras (TW) como variável independente e o crescimento anual das vendas (CRESC), alavancagem (AL), tamanho da empresa (TAM) e retorno sobre os ativos (ROA) como variáveis de controle. Essas variáveis foram extraídas da base de dados Thomson Reuters ${ }^{\circledR}$ e do Twitter. Demonstram-se na Tabela 2, detalhadamente, as variáveis analisadas no estudo.

Tabela 2. Variáveis utilizadas no estudo

\begin{tabular}{|c|c|c|c|c|}
\hline \multicolumn{2}{|c|}{ Variáveis/ Definição } & Fórmula & Coleta & Autores \\
\hline \multicolumn{5}{|c|}{ Variável dependente - Desempenho de mercado (DES) } \\
\hline DES & Retorno anormal & $\begin{array}{l}\text { Retorno da ação }{ }_{t}-\text { Retorno } \\
\text { médio do setor }\end{array}$ & Thomson $^{\circledR}$ & $\begin{array}{l}\text { Yu et al 2013; Miller \& Skinner, } \\
\text { 2015; Jung et al. (2018); Cao et al. } \\
\text { (2018) }\end{array}$ \\
\hline \multicolumn{5}{|c|}{ Variável independente } \\
\hline TW & Tweets & $\begin{array}{l}\text { Número de publicações } \\
\text { referentes a informações } \\
\text { financeiras }\end{array}$ & Twitter & $\begin{array}{l}\text { Lee et al. (2015); Manetti, Belluci } \\
\text { e Bagnoli (2017); Jung et al. } \\
\text { (2018). }\end{array}$ \\
\hline \multicolumn{5}{|c|}{ Variáveis de controle } \\
\hline CRESC & $\begin{array}{l}\text { Crescimento das } \\
\text { vendas }\end{array}$ & $\frac{\left(\text { Receita }_{t}-\text { Receita }_{t-1}\right)}{\text { Receita }_{t-1}}$ & & $\begin{array}{c}\text { Lee et al. (2015); Rodriguez et al. } \\
\text { (2015); Ainin et al. (2015); Jung et } \\
\text { al. (2018) }\end{array}$ \\
\hline $\mathrm{AL}$ & Alavancagem & $\frac{\text { Passivo total }}{\text { Ativo total }}$ & & Zhang (2015); Jung et al. (2018) \\
\hline TAM & Tamanho & Log do ativo total & Thomson $^{\circledR}$ & $\begin{array}{c}\text { Lee et al. (2015); Zhang (2015); } \\
\text { Jung et al. (2018); Parveen et al. } \\
\text { (2016). }\end{array}$ \\
\hline ROA & $\begin{array}{l}\text { Retorno sobre os } \\
\text { ativos }\end{array}$ & $\frac{\text { EBIT }}{\text { Ativo total }}$ & & $\begin{array}{l}\text { Fang \& Peress, 2009; Zhang } \\
\text { (2015); Ainin et al. (2015) Jung et } \\
\text { al. (2018); Cao et al. (2018) }\end{array}$ \\
\hline
\end{tabular}

Fonte: dados da pesquisa.

Quanto à análise dos dados, primeiramente realizou-se a winsorização das variáveis. Na sequência, realizou-se o teste de normalidade Shapiro-Wilk, o qual demonstrou que os dados não são normais $(Z=13,326$; $\mathrm{z}$ $<0,000)$. Assim, efetuou-se o teste de médias Mann Whitney e as correlações. Por fim, com o intuito de atender ao objetivo do estudo, foram operacionalizadas regressões OLS com erros padrão robustos e controle de efeitos fixos de setor e ano, por meio do software STATA. O modelo empírico apresenta-se na equação a seguir.

$$
D E S_{i t}=\beta_{0}+\beta_{1} T W_{i t}+\beta_{2} V C_{i t}+\text { Efeitosfixos_setor }+ \text { Efeitosfixos_ano }+\varepsilon
$$

A regressão foi operacionalizada com e sem as variáveis de controle (VC), com o propósito de evidenciar a relação direta do Twitter (TW) no desempenho organizacional, bem como quando incluída as variáveis de controle. Justifica-se a realização de regressão robusta pois o teste White apresentou-se significativo $(\mathrm{P}=456,12 ; \mathrm{p}<0,000), \mathrm{o}$ que indica a presença de heterocedasticiadade. Apesar da não normalidade dos dados, esse pressuposto da regressão linear OLS foi relaxado ao considerar o Teorema Central do Limite, devido ao número de observaçãos. Além disso, testou-se a multicolinearidade entre as variáveis, pelo teste Variance Inflation Factor (VIF), e a autocorrelação dos resíduos, pelo teste Durbin Watson, cujos resultados estão apresentados na Tabela 5. 


\section{APRESENTAÇÃO E ANÁLISE dOS RESULTADOS}

Inicialmente apresenta-se a estatística descritiva das variáveis e o teste de médias (Mann Whitney), a fim de verificar se há diferenças significativas entre as empresas que utilizam ou não as mídias sociais para publicar informações financeiras (tweets) e o desempenho de mercado. A estatística descritiva é demonstrada na Tabela 3.

Tabela 3. Estatística descritiva das variáveis

\begin{tabular}{lcccccccc}
\hline & \multicolumn{2}{c}{ Empresas com tweets } & \multicolumn{2}{c}{ Empresas sem tweets } & \multicolumn{2}{c}{ Amostra total } & \multicolumn{2}{c}{$\begin{array}{c}\text { Teste de Média Mann } \\
\text { Whitney }\end{array}$} \\
\hline & Média & $\begin{array}{l}\text { Desvio } \\
\text { Padrão }\end{array}$ & Média & $\begin{array}{c}\text { Desvio } \\
\text { Padrão }\end{array}$ & Média & $\begin{array}{c}\text { Desvio } \\
\text { Padrão }\end{array}$ & Z & Sig. \\
\hline DES & 0,00251 & 0,059 & $-0,00606$ & 0,054 & $-0,00519$ & 0,055 & $-2,114$ & 0,412 \\
CRESC & 0,087 & 0,193 & 0,059 & 0,450 & 0,062 & 0,431 & $-1,776$ & $0,034^{* *}$ \\
AL & 0,588 & 0,186 & 0,767 & 0,655 & 0,749 & 0,626 & $-11,180$ & $0,076^{*}$ \\
TAM & 22,509 & 1,594 & 20,321 & 1,741 & 20,545 & 1,849 & $-1,097$ & $0,000^{* * *}$ \\
ROA & 0,059 & 0,051 & 0,028 & 0,155 & 0,032 & 0,148 & $-0,820$ & 0,273 \\
\hline
\end{tabular}

Fonte: dados da pesquisa.

Nota: Legenda: DES. Retorno Anormal; CRESC. Crescimento; AL. Alavancagem; TAM. Tamanho; ROA. Retorno sobre os ativos. Níveis de significância: * $\mathrm{p}<0,1, * * \mathrm{p}<0,05, * * * \mathrm{p}<0,01$.

Observa-se na Tabela 3 que o crescimento das empresas demonstrou diferença estatisticamente significante ao nível de $5 \%$, o que sugere que empresas que publicam tweets de informações financeiras apresentam maior crescimento das vendas. Para Cao et al. (2018), o uso de mídias sociais ajuda a capturar clientes e impulsionar as vendas, o que aumenta a receita e a participação de mercado.

A variável alavancagem demonstrou diferença estatisticamente significante ao nível de $10 \%$, conforme o estudo de Jung et al. (2018), os quais evidenciaram que empresas com maior alavancagem tendem a usar menos a mídia social. Além disso, as empresas que divulgam notícias financeiras no Twitter tendem a ser maiores, o tamanho da empresa apresentou diferença estatisticamente significante ao nível de 1\%.

As variáveis retorno anormal e restorno sobre os ativos não apresentaram diferença estatisticamente significativa. Entretanto, observa-se que ambas as médias são maiores para as empresas que publicam tweets sobre informações financeiras. Destaca-se que as empresas que publicam apresentaram um retorno anormal médio positivo e as que não publicam negativo, de $(0,25 \%)$ e $(-0,60 \%)$, respectivamente.

Em seguida, na Tabela 4 apresenta-se a Correlação de Spearman no triângulo superior direito e a correlação de Pearson no triâgulo inferior esquerdo.

Tabela 4. Correlação de Spearman e Pearson

\begin{tabular}{lcccccc}
\hline Variável & DES & TW & CRESC & AL & TAM & ROA \\
\hline DES & 1 & $-0,005$ & $0,146^{* * *}$ & $-0,107 * * *$ & $0,071^{* *}$ & $0,175^{* * *}$ \\
TW & $0,103 * * *$ & 1 & $0,048^{* * *}$ & $-0,004 * * *$ & $0,234 * * *$ & 0,039 \\
CRESC & 0,044 & 0,011 & 1 & $-0,033^{* * * *}$ & $0,205^{* * *}$ & $0,384^{* * *}$ \\
AL & $-0,085 * * *$ & $-0,045$ & $-0,027$ & 1 & 0,022 & $-0,214 * * *$ \\
TAM & $0,097 * * *$ & $0,234 * * *$ & $0,128^{* * *}$ & $-0,267$ & 1 & $0,262^{* * *}$ \\
ROA & $0,076^{* * *}$ & 0,038 & $0,162 * * *$ & $-0,481 * * *$ & $0,306 * * *$ & 1 \\
\hline
\end{tabular}

Fonte: dados da pesquisa.

Nota: Legenda: DES. Retorno Anormal; CRESC. Crescimento; AL. Alavancagem; TAM. Tamanho; ROA. Retorno sobre os ativos. Níveis de significância: $* \mathrm{p}<0,1, * * \mathrm{p}<0,05, * * * \mathrm{p}<0,01$.

Conforme observado na Tabela 4, na correlação de Spearman, o retorno anormal (DES) apresenta uma correlação significativa e positiva com o crescimento da empresa $(0,146)$, o tamanho $(0,071)$ e o retorno sobre os ativos $(0,175)$ e está correlacionado negativamente com a alavancagem $(-0,107)$, o que está alinhado com a predição teórica. O número de tweets se correlaciona de maneira significativa e positiva com o crescimento $(0,48)$ e o tamanho da empresa $(0,234)$ e negativamente com a alavancagem $(-0,004)$. Na correlação de Pearson destacase a correlação positiva e significativa entre o desempenho de mercado e a publicação de tweets ao nível de $1 \%$. 
Para a realização do teste da hipótese, realizou-se regressões lineares múltiplas (OLS), com erros padrão robustos e com controle de efeitos fixos de setor e ano. Os resultados estão resumidos na Tabela 5.

Tabela 5. Resultado regressão uso corporativo das mídias sociais e desempenho organizacional

\begin{tabular}{lccccc}
\hline \multirow{2}{*}{ Variáveis } & \multirow{2}{*}{ Sinal previsto } & \multicolumn{3}{c}{ Variavél dependente: Retorno anormal } \\
\cline { 3 - 6 } & & Coeficiente & Estatística $t$ & Coeficiente & Estatística $t$ \\
\hline Constante & +- & $-0,211$ & $(-0,22)$ & $-1,864$ & $(-0,74)$ \\
TW & + & $0,396^{* *}$ & $(2,04)$ & $0,340^{*}$ & $(1,70)$ \\
CRESC & + & - & - & 0,236 & $(0,47)$ \\
ALAV & - & - & - & $-0,453^{* *}$ & $(-1,94)$ \\
TAM & + & - & - & 0,099 & $(0,94)$ \\
ROA & + & - & - & 1,273 & $(0,91)$ \\
\hline Efeitos fixos setor e ano & & Sim & & Sim \\
Significância do modelo & & $0,000^{* * *}$ & & $0,000^{* * *}$ \\
R & & 4,56 & & 5,35 \\
R ajustado & 3,36 & & 3,81 \\
VIF & & 1,08 & & $1,09-1,49$ \\
DW & & 1,828 & & 1,827 \\
$\mathrm{~N}$ & & 1.125 & & 1.125 \\
\hline
\end{tabular}

Fonte: dados da pesquisa.

Nota: Legenda: VIF = Variance Inflation Factor; DW = Durbin-Watson; $\mathrm{N}=$ número de observações. Níveis de significância: * p<0,1, ** $\mathrm{p}<0,05, * * * \mathrm{p}<0,01$.

No que se refere à relação direta entre o uso corporativo de mídias sociais, mensurado por meio do número de publicações sobre informações financeiras, e o desempenho de mercado, observado por meio do retorno anormal das ações, verificou-se uma relação positiva e significativa ao nível de $5 \%$. Ao operacionalizar o número de informações publicadas com as demais variáveis de controle, a relação positiva e significativa entre o número de publicações e o retorno anormal é confirmada, entretanto ao nível de $10 \%$.

Ao comparar a média do retorno anormal (DES) de -0,519\% (Tabela 3 ) e o coeficiente de 0,396 (Tabela 5) da variável TW, nota-se uma redução média de $0,76 \%(0,396:-0,519 \%)$ do retorno anormal negativo em empresas que utilizam o Twitter. Quando avaliado em conjunto, a variável independente e as variáveis de controle, em termos econômicos, ao considerar o coeficiente do TW (0,340 Tabela 5) e a média do retorno anormal $(-0,519)$, percebese uma redução média de $0,65 \%(0,340:-0,519 \%)$ no retorno anormal negativo, por meio do uso corporativo de mídia social para divulgar informações financeiras.

Isso sugere que o uso corporativo dessa mídia social para publicar tweets está associado ao desempenho de mercado das empresas brasileiras analisadas, mais especificamente, as empresas com tweets de informações financeiras publicados apresentam um retono anormal acima da média das demais empresas do setor. Pode-se depreender deste resultado que empresas mais lucrativas divulgam suas informações pelo Twitter, possivelmente a fim de dar maior publicidade aos seus resultados positivos, o que acaba atraindo novos investidores, alavancando seu desempenho de mercado.

Em relação às variáveis de controle, percebe-se que a alavancagem apresentou relação negativa e significativa com o desempenho organizacional, o que sugere que quanto maior o endividamento da empresa, menor será o retorno anormal das ações. Esse achado corrobora o estudo de Chen e Zhao (2006), que demonstra que o endividamento possui relação negativa com o desempenho organizacional.

Dessa maneira, não se pode rejeitar a $\mathrm{H}_{1}$. De modo geral, infere-se que a utilização de mídias sociais para fins corporativos, mais especificamente o uso do Twitter para publicar informações financeiras, pode contribuir para o desempenho de mercado, no que tange ao retono anormal das ações. Esse achado corrobora estudos anteriores que relataram que as mídias sociais afetam e beneficiam a organização de várias formas, como por meio da divulgação de notícias corporativas (Zhang, 2015), ao disponibilizar informações de forma rápida, consistente e contínua aos seus clientes, investidores e partes interessadas, além de minimizar informações negativas (Jung et al., 2018; Lee et al., 2015). 
A fim de dar maior confiabilidade aos resultados, foi realizado um teste adicional acerca da relação entre o uso corporativo das mídias sociais para publicação de informações financeiras e o desempenho de mercado. Para isso, utilizou-se o retorno das ações (calculado por meio do preço da ação no período $t$ subtraído do preço da ação no período $t-1$, dividido pelo preço da ação no período $t-1$ ) como variável dependente. A Tabela 6 apresenta os resultados do teste de robustez.

Tabela 6. Teste de robustez

\begin{tabular}{lccccc}
\hline \multirow{2}{*}{ Variáveis } & \multirow{2}{*}{ Sinal previsto } & \multicolumn{3}{c}{ Variavél dependente: Retorno das ações } \\
\cline { 3 - 6 } & & Coeficiente & Estatística $t$ & Coeficiente & Estatística $t$ \\
\hline Constante & +- & 0,130 & $(0,14)$ & $-1,688$ & $(-0,70)$ \\
TW & + & $0,384^{* *}$ & $(1,96)$ & $0,324^{*}$ & $(1,60)$ \\
CRESC & + & - & - & 0,251 & $(0,50)$ \\
ALAV & - & - & - & $-0,473 *$ & $(-1,90)$ \\
TAM & + & - & - & 0,107 & $(1,05)$ \\
ROA & + & - & - & 1,265 & $(0,72)$ \\
\hline Efeitos fixos setor e ano & & Sim & & Sim \\
Significância do modelo & & $0,000^{* * *}$ & & $0,000^{* * *}$ \\
$\mathrm{R}^{2}$ & & 41,56 & & 42,06 \\
$\mathrm{R}^{2}$ ajustado & & 40,82 & & 41,12 \\
VIF & & 1,08 & & $1,09-1,49$ \\
DW & & 1,819 & & 1,819 \\
$\mathrm{~N}$ & & 1.125 & & 1.125 \\
\hline
\end{tabular}

Fonte: dados da pesquisa.

Nota: Legenda: VIF = Variance Inflation Factor; DW = Durbin-Watson; $\mathrm{N}=$ número de observações. Níveis de significância: * p<0,1, ** $\mathrm{p}<0,05, * * * \mathrm{p}<0,01$.

De acordo com os resultados observados na Tabela 6, constata-se que a varíavel de interesse, publicações de tweets, apresentou relação significativa com o retorno das ações. Pode-se inferir, por meio desse resultado, que a publicação de informações financeiras no Twitter tem efeito também sobre o desempenho de mercado, conforme observado na Tabela 5.

Os resultados deste estudo contribuem com a literatura sobre o uso corporativo das mídias sociais, ao confirmar as inferências de que a utilização das mídias sociais possui relação positiva com o desempenho da empresa (Ainin et al. 2015; Arnaboldi \& Coget, 2016; Cao et al., 2018; Parveen et al., 2016; Shuai \& Wu, 2011), evidenciado por meio do retorno anormal das ações.

Para Garcia-Morales et al. (2018), o uso corporativo de mídias sociais possibilita que a empresa desenvolva competências e adquira conhecimento sobre seus clientes, o que promove o desempenho organizacional. Este estudo contribui ao demonstrar, por meio de resultados empíricos, que a publicação de informações no Twitter, o qual é caracterizado por uma comunicação rápida, sem intermediários e multidirecional, pode potencializar os resultados positivos já obtidos.

Cabe destacar que as palavras-chaves utilizadas para busca de tweets com informações financeiras presumem informações positivas, referentes a resultados positivos obtidos pela empresa. Nesse sentido, o intuito de divulgar informações com esse conteúdo em mídias sociais teria como objetivo dar maior visibilidade a essas informações e, dessa forma, influenciar a tomada de decisão dos interessados na empresa. Isso fica evidenciado neste estudo, ao se observar maiores retornos anormais.

Apesar de a informação divulgada no Twitter não se caracterizar como nova, o fato da organização disponibilizá-la em mídia social possibilita uma interação, troca de informações multidirecionais, compartilhamento de opiniões entre os próprios usuários da mídia, o que pode refletir na tomada de decisão dos investidores. Assim, o uso corporativo de mídia social, atrelado à divulgação de informações financeiras, demonstra que o caráter tecnológico dessa ferramenta proporciona benefícios à organização, como maior visibilidade e melhor desempenho de mercado. 


\section{CONSIDERAÇÕES FINAIS}

Esta pesquisa teve por objetivo investigar relação entre o uso corporativo de mídias sociais e o desempenho de mercado em empresas brasileiras, no período de cinco anos (2013 a 2017). Os resultados demonstraram que o uso corporativo do Twitter para divulgar informações financeiras está relacionado com o retorno anormal das ações, o que não permite rejeitar a hipótese $H_{1}$.

Isso pode sugerir que as divulgações no Twitter influenciam o comportamento de investidores e analistas e, consequentemente, o retorno anormal das ações. Ainda que o número de empresas brasileiras que divulgaram informações corporativas no Twitter no período analisado tenha sido reduzido, é possível notar um aumento desse tipo de divulgação por parte das empresas nesse mesmo período. Como o uso das mídias sociais parece ser dinâmico, é possível que essa mesma análise em períodos mais recentes aponte resultados ainda mais significativos.

Há que se considerar, no entanto, que os termos buscados para publicações no Twitter sugerem que as informações publicadas sejam positivas. Esse fato pode indicar que empresas que já possuem resultados positivos, relacionados à crescimento, receitas, lucros, entre outros, ao divulgá-los no Twitter, podem maximizar o seu desempenho de mercado.

Os resultados deste estudo contribuem para a literatura que examina os comportamentos estratégicos das empresas sobre a utilização do Twitter para a divulgação de informações. Além disso, os achados evidenciam benefícios reais obtidos com o uso das mídias sociais, ao demonstrar que o uso corporativo do Twitter pode influenciar os participantes do mercado de capitais e, consequentemente, melhorar o desempenho de mercado da organização. Tal evidência pode motivar a alta gerência das empresas a fornecer suporte inicial e contínuo para o uso de mídias sociais nas organizações.

A presente pesquisa apresenta limitações, tais como a impossibilidade da generalização dos resultados, visto que foram analisadas apenas as empresas listadas na B3 (Brasil, Bolsa e Balcão) com informações disponíveis na base de dados Thomson Reuters Eikon, no período de 2013 a 2017. Além disso, utilizou-se como mídia social apenas o Twitter, sendo que outras poderiam ser utilizadas, o que também representa uma lacuna para pesquisas futuras.

\section{REFERÊNCIAS}

Agostino, D., \& Sidorova, Y. (2017). How social media reshapes action on distant customers: some empirical evidence. Accounting, Auditing \& Accountability Journal, 30(4), 777-794. DOI: https://oi.org/10.1108/ AAAJ-07-2015-2136

Ainin, S., Parveen, F., Moghavvemi, S., Jaafar, N. I., \& Shuib, N. L. M. (2015). Factors influencing the use of social media by SMEs and its performance outcomes. Industrial Management \& Data Systems.

Arias, M., Arratia, A., \& Xuriguera, R. (2014). Forecasting with Twitter data. ACM Transactions on Intelligent Systems and Technology, 5(1), 1-24. DOI: https://doi.org/10.1145/2542182.2542190

Arnaboldi, M., Busco, C., \& Cuganesan, S. (2017). Accounting, accountability, social media and big data: revolution or hype?. Accounting, Auditing \& Accountability Journal, 30(4), 762-776. DOI: https://doi. org/10.1108/AAAJ-03-2017-2880

Arnaboldi, M., \& Coget, J. F. (2016). Social media and business. Organizational Dynamics, 1(45), 47-54.

Bonson, E., \& Ratkai, M. (2013). A set of metrics to assess stakeholder engagement and social legitimacy on a corporate Facebook page. Online Information Review, 37(5), 787-803. DOI: https://doi.org/10.1108/OIR-032012-0054

Cao, Y., Ajjan, H., Hong, P., \& Le, T. (2018). Using social media for competitive business outcomes: An empirical study of companies in China. Journal of Advances in Management Research, 15(2), 211-235. DOI: https:// doi.org/10.1108/JAMR-05-2017-0060

Chen, L., \& Zhao, X. (2006). On the relation between the market-to-book ratio, growth opportunity, and leverage ratio. Finance Research Letters, 3(4), 253-266. DOI: https://doi.org/10.1016/j.frl.2006.06.003

Fang, L., \& Peress, J. (2009). Media coverage and the crosssection of stock returns. The Journal of Finance, 64(5), 2023-2052. DOI: https://doi.org/10.1111/j.1540-6261.2009.01493.x 
Ferrer, E., Bousoño, C., Jorge, J., Lora, L., Miranda, E., \& Natalizio, N. (2013). Enriching social capital and improving organizational performance in the age of social networking. International Journal of Information, Business and Management, 5(2), 94.

Frankel, R., Johnson, M., \& Skinner, D. J. (1999). An empirical examination of conference calls as a voluntary disclosure medium. Journal of Accounting Research, 37(1), 133-150. DOI: https://doi.org/10.2307/2491400

Garcia-Morales, V. J., Martín-Rojas, R., \& Lardón-López, M. E. (2018). Influence of social media technologies on organizational performance through knowledge and innovation. Baltic Journal of Management. DOI: https:// doi.org/10.1108/BJM-04-2017-0123

Harquail, C. (2011). Recreating reputation through authentic interaction: Using social media to connect with individual stakeholders, in Burke, R., Martin, G., and Cooper, C., (Eds.), Corporate reputation. Managing opportunities and threats. Gower Publishing, Burlington, VT, 245-263.

Harris, A. L., \& Rea, A. (2009). Web 2.0 and virtual world technologies: A growing impact on IS education. Journal of Information Systems Education, 20(2), 137.

He, W., Wang, F. K., \& Akula, V. (2017). Managing extracted knowledge from big social media data for business decision making. Journal of Knowledge Management, 21(2), 275-294. DOI: https://doi.org/10.1108/JKM07-2015-0296

Jung, M. J., Naughton, J. P., Tahoun, A., \& Wang, C. (2018). Do firms strategically disseminate? Evidence from corporate use of social media. The Accounting Review. DOI: https://doi.org/10.2308/accr-51906

Kaplan, A. M., \& Haenlein, M. (2010). Users of the world, unite! The challenges and opportunities of Social Media. Business horizons, 53(1), 59-68. DOI: https://doi.org/10.1016/j.bushor.2009.09.003

Kim, E. H., \& Youm, Y. N. (2017). How do social media affect analyst stock recommendations? Evidence from S\&P 500 electric power companies' Twitter accounts. Strategic Management Journal, 38(13), 2599-2622. DOI: https://doi.org/10.1002/smj.2678

Lee, L.F., Hutton, A. P. \& Shu, S. (2015). The Role of Social Media in the Capital Market: Evidence from Consumer Product Recalls. Journal of Accounting Research 53, 367-404. DOI: https://doi.org/10.1111/1475679X.12074

Lodhia, S., \& Stone, G. (2017). Integrated reporting in an internet and social media communication environment: conceptual insights. Australian Accounting Review, 27(1), 17-33. DOI: https://doi.org/10.1111/auar.12143

Manetti, G., Bellucci, M., \& Bagnoli, L. (2017). Stakeholder engagement and public information through social media: A study of Canadian and American Public Transportation Agencies. The American Review of Public Administration, 47(8), 991-1009. DOI: https://doi.org/10.1177/0275074016649260

Miller, G. S., \& Skinner, D. J. (2015). The evolving disclosure landscape: How changes in technology, the media, and capital markets are affecting disclosure. Journal of Accounting Research, 53(2), 221-239. DOI: https:// doi.org/10.1111/1475-679X.12075

Paniagua, J., \& Sapena, J. (2014). Business performance and social media: Love or hate?. Business horizons, 57(6), 719-728. DOI: https://doi.org/10.1016/j.bushor.2014.07.005

Parveen, F., Jaafar, N. I., \& Ainin, S. (2016). Social media's impact on organizational performance and entrepreneurial orientation in organizations. Management Decision, 54(9), 2208-2234. DOI: https://doi. org/10.1108/MD-08-2015-0336

Rodriguez, M., Peterson, R. M., \& Ajjan, H. (2015). CRM/social media technology: impact on customer orientation process and organizational sales performance. Ideas in Marketing: Finding the New and Polishing the Old. 636-638

Scott, S. V. \& Orlikowski, W. J. (2012). Reconfiguring relations of accountability: Materialization of social media in the travel sector. Accounting, Organizations and Society, 37(1), 26-40. DOI: https://doi.org/10.1016/j. aos.2011.11.005

Shuai, J. J. \& Wu, W. W. (2011), Evaluating the influence of E-marketingon hotel performance by DEA and grey entropy. Expert Systems with Applications, 38(7), 8763-8769. DOI: https://doi.org/10.1016/j.eswa.2011.01.086

Smits, M., \& Mogos, S. (2013). The Impact Of Social Media On Business Performance. ECIS. p. 125. 
Wetzstein, B., Leitner, P., Rosenberg, F., Dustdar, S., \& Leymann, F. (2011). Identifying influential factors of business process performance using dependency analysis. Enterprise Information Systems, 5(1), 79-98. DOI: https://doi.org/10.1080/17517575.2010.493956

Yu, Y., Duan, W., \& Cao, Q. (2013). The impact of social and conventional media on firm equity value: a sentiment analysis approach. Decision Support Systems, 55(4) 919-926. DOI: https://doi.org/10.1016/j.dss.2012.12.028

Zhang, J. (2015). Voluntary information disclosure on social media. Decision Support Systems, 73, 28-36. DOI: https://doi.org/10.1016/j.dss.2015.02.018

\section{Como citar este artigo}

Giordani, M. da S.; Lunardi, M. A.; \& Klann, R. C. (2020). Uso corporativo de mídias sociais e o desempenho de mercado. Revista de Contabilidade e Organizações, 14:e169560. DOI: http://dx.doi. org/10.11606/issn.1982-6486.rco.2020.169560 